\title{
Do Cardiometabolic Risk Factors Relative Risks Differ for the Occurrence of Ischemic Heart Disease and Stroke?
}

\author{
Samaneh Aalami Harandi ${ }^{1}$; Nizal Sarrafzadegan ${ }^{2,}$; Masoumeh Sadeghi ${ }^{3}$; Mohammad \\ Talaei ${ }^{2}$; Mino Dianatkhah ${ }^{1}$; Shahram Oveisgharan ${ }^{2}$; Ali Pourmoghaddas ${ }^{4}$; Asma Salehi ${ }^{4}$; \\ Zohre Sedighifard $^{4}$ \\ ${ }^{1}$ Hypertension Research Center, Cardiovascular Research Institute, Isfahan University of Medical Sciences, Isfahan, IR Iran \\ 2 Isfahan Cardiovascular Research Center, Cardiovascular Research Institute, Isfahan University of Medical Sciences, Isfahan, IR Iran \\ ${ }^{3}$ Cardiac Rehabilitation Research Center, Cardiovascular Research Institute, Isfahan University of Medical Sciences, Isfahan, IR Iran \\ ${ }^{4}$ Department of Cardiology, School of Medicine, Isfahan University of Medical Sciences, Isfahan, IR Iran \\ ${ }^{*}$ Corresponding author: Nizal Sarrafzadegan, Isfahan Cardiovascular Research Center, Cardiovascular Research Institute, Isfahan University of Medical Sciences, Isfahan, IR Iran. \\ E-mail: nsarrafzadegan@gmail.com \\ Received: June 13, 2015; Accepted: July 19, 2015
}

\begin{abstract}
Background: The effects of the risk factors of ischemic heart disease (IHD) and stroke on the occurrence of these diseases differ between different populations.

Objectives: To study the difference in the effects of different cardiovascular (CVD) risk factors on the incidence of IHD and stroke in an Iranian adult population.

Patients and Methods: The Isfahan Cohort Study (ICS) is a longitudinal study that followed up 6323 subjects older than 35 years with no history of CVD since 2001. Of the original sample, only 5431 participants were contacted and followed up until 2011. The end points were the occurrence of IHD (defined as fatal and non-fatal myocardial infarction, unstable angina, and sudden cardiac death) and stroke. After 10 years of follow-up, 564 new cases of IHD and 141 new cases of stroke were detected. The relative risks(RRs) of cardiometabolic risk factors such as hypertension, diabetes, hypercholesterolemia, hypertriglyceridemia, high low-density lipoprotein cholesterol (LDL-C) level, low high-density lipoprotein cholesterol (HDL-C) level, current smoking, obesity, high waist-to-hip ratio, family history of CVD, and metabolic syndrome were compared between IHD and stroke patients. The ratio of relative risks (RRR) was calculated for comparing two RRs and estimated adjusted RRR was calculated by using generalized linear regression with a log link and binomial distribution.

Results: The RRs of the occurrence of IHD and stroke in diabetic patients were 1.94 and 3.26, respectively, and the difference was statistically different $(\mathrm{P}=0.016)$. The RR of high LDL-C was significantly higher for IHD than for stroke $(\mathrm{P}=0.045)$, while all the other risk factors showed similar RRs for IHD and stroke, with no significant difference in their RRR, including hypertension. Diabetes and hypertension had the highest RRs for IHD, followed by diabetes, metabolic syndrome, and hypertension for stroke.

Conclusions: The effect of diabetes mellitus on stroke was more significant than on IHD, and the effect of high LDL-C level was more significant on IHD than on stroke, other risk factors, including hypertension, have similar RRs for IHD and stroke. Health care professionals need more training regarding the RRs of these risk factors in the Iranian society, and health decision makers should consider it in their future policies.
\end{abstract}

Keywords: Cardiovascular Diseases; Risk Factors; Stroke; Myocardial Ischemia; Cohort Studies

\section{Background}

The increasing prevalence of chronic metabolic diseases in the world, especially in developing countries, poses increasing threats at a global level. The World Health Organization views cardiovascular diseases (CVD) as an important cause of death in low- and middle-income countries (1), according to which about $76 \%$ of $25,000,000$ deaths in developing countries by 2020 are caused by CVD. In the Middle Eastern countries, especially in the 2 recent decades, an outbreak of chronic diseases that cause high morbidity and mortality, including diabetes mellitus, hypertension, CVD, and stroke occurred (2-4). In recent years, Iran faced an increasing prevalence of CVD and metabolic diseases due to economic growth, improvement in health care, and a subsequent decrease in infectious diseases, aging, and changes in lifestyle (5). Although the risk factors of ischemic heart disease
(IHD) and stroke are common in some properties, their effects on the occurrence of these diseases differ. For instance, hypertension exerts a significant effect on stroke (6), while dyslipidemia exerts a significant effect on IHD $(7,8)$. In addition, their effects differ between different populations. Metabolic risk factors such as diabetes mellitus, dyslipidemia, and hypertension, along with other factors such as age, sex, smoking, and obesity (body mass index [BMI] and proportion of waist to hip) are interrelated with heart diseases and stroke (9-14).

A large number of studies have concentrated on the risk factors of heart diseases and stroke. However, they could not completely cover and evaluate the relative risk (RR) of each factor on the incidence of IHD and stroke within a prospectively long-term follow-up in the Middle Eastern countries, including Iran. Generally, a small number

Copyright (C) 2016, Rajaie Cardiovascular Medical and Research Center, Iran University of Medical Sciences. This is an open-access article distributed under the terms of the Creative Commons Attribution-NonCommercial 4.0 International License (http://creativecommons.org/licenses/by-nc/4.0/) which permits copy and redistribute the material just in noncommercial usages, provided the original work is properly cited. 
of studies examined the strength of the relationship between metabolic risk factors and CVD and stroke altogether. Discussions over their differences can contribute to a proper understanding of the baseline mechanisms of these diseases and highlight the effect of certain interventions on long-term outcomes of risk factors to make proper treatment strategies (15-17). Meanwhile, these risk factors affect different races and populations differently.

\section{Objectives}

The present study aimed to evaluate and compare between the RRs of cardiometabolic risk factors of the incidence of IHD and stroke in an Iranian adult population who participated in the Isfahan Cohort Study (ICS) (18).

\section{Patients and Methods}

ICS is a prospective cohort study started in January 2001 and is still ongoing in urban and rural areas of Isfahan, Najafabad, and Arak in Central Iran (18). The population, initially 6504 , consisted of subjects older than 35 years constituted the baseline sample of the Isfahan Healthy Heart Program (19). From this study, 181 subjects with a history of myocardial infarction (MI) stroke or any other CVD were excluded. Finally, the study evaluated 6323 participants who were recruited in 2001 in the ICS, of whom 5431 participants had at least one follow-up visit until 2011. The whole design, methods, and sampling were reported elsewhere (18). Briefly, demographic variables such as sex, age, education, history of smoking, diabetes, hypertension, and dyslipidemia were determined. Cardiometabolic variables such as fasting blood glucose (FBG) and 2-hour postprandial (2 hpp) total cholesterol, high-density lipoprotein cholesterol (HDL-C), low-density lipoprotein cholesterol (LDL-C), and triglyceride (TG) levels; systolic and diastolic blood pressure; BMI; and waist circumference were measured and recorded in 2001. Then, in 5-year intervals, blood sampling, and physical examination were repeated, while in 2-year intervals, the data of the occurrence of fatal and non-fatal CVDs or hospitalization were recorded by a trained nurse by phone calls. With regard to hospital stay or death, the medical documents of the patients were analyzed in the respective hospital by a general physician, and the occurrence of CVDs, such as fatal and non-fatal IHD, and fatal and non-fatal stroke were recorded. Although the in-hospital diagnoses by the physicians were taken into account, the final diagnosis was confirmed by 3 cardiologists and 1 neurologist. IHD definition includes fatal and non-fatal acute MI, unstable angina, and sudden cardiac death. Acute MI was defined as the presence of at least two of the following criteria (18): typical chest pain lasting more than $30 \mathrm{~min}$ utes of more than 0.1 MW of ST elevation in at least two contagious leads and positive cardiac biomarkers, including troponin I or T or creatine kinase-MB fraction. Unstable angina was defined as typical chest pain or chest discomfort at rest or at low level activity, which lasts at least $20 \mathrm{~min}$ utes, representing a change in the usual pattern of angina, or as pain causing a frank pain along with new or dynamic
ST-T changes in two adjacent electrocardiographic leads. Sudden cardiac death was described as an unpredictable death outside the hospital that happens in less than 1 hour after symptoms arise. Death without existence of any data regarding symptoms is categorized as death of unknown cause.

Stroke is clinically defined as a neurological focal deficit with sudden onset, lasting at least 24 hours, and is probably caused by vascular parameters. Metabolic syndrome was defined according to the national cholesterol education programme adult treatment panel III (20) and existence of three or more of the following criteria: fasting TG level $\geq 150 \mathrm{mg} / \mathrm{dL}$; HDL-C level $<40 \mathrm{mg} / \mathrm{dL}$ in men and $<50$ in women; fasting glucose level $\geq 110 \mathrm{mg} / \mathrm{dL}$; blood pressure $\geq 130 / 85 \mathrm{mmHg}$ or receiving antihypertensive medications; and a waist circumference $>102 \mathrm{~cm}$ in men and $>88 \mathrm{~cm}$ in women.

LDL-Clevel $\geq 130 \mathrm{mg} /$ dLortotalcholesterollevel $\geq 200 \mathrm{mg}$ $\mathrm{dL}$, or TG level $\geq 150 \mathrm{mg} / \mathrm{dL}$ or HDL-C $<40 \mathrm{mg} / \mathrm{dL}$ in men or $<50 \mathrm{mg} / \mathrm{dL}$ in women were classified as dyslipidemia. Diabetes mellitus was defined if FBG level $\geq 126 \mathrm{mg} / \mathrm{dL}$ or $2 \mathrm{hpp}$ $\geq 200$, or if the patient was receiving antidiabetic agents. ObesitywasasaBMI $\geq 30 \mathrm{~kg} / \mathrm{m}^{2}$. Waist-to-hip ratio(WHR) $\geq$ 0.95 in men and $\geq 0.8$ in women was considered as a high WHR. Those who had blood pressures $\geq 140 / 90 \mathrm{mmHg}$ or patients receiving antihypertensive drugs were considered as hypertensive. A family history of premature CVD in firstdegree relatives was defined as the occurrence of cardiovascular diseases before the age of 55 years for men and 65 years for women. Smoking means current smoking.

\subsection{Statistical Analysis}

All the collected data of the patients were managed and analyzed by using the Stata IC 11.0 software (Stata Corp LP, TX, USA). Data are expressed as mean \pm SD for continuous variables and number (percentage) for categorical variables. The chi-square test was used for the comparison of proportions and categorical variables, and analysis of variance was used for the comparison of quantitative variables. The incidence rate was the number of new cases during the study period divided by the sum of the person-time of the at-risk population. Ratio of relative risks (RRR) was calculated for comparing two RRs (21). Adjusted RR was calculated by using generalized linear regression with a log link and binomial distribution (22). A P $<0.05$ was considered statistically significant.

\section{Results}

Among the original 6323 participants in the ICS, 5431 had at least one follow-up visit between 2001 and 2011 . In this period, 705 CVD events occurred, including 564 IHD and 141 strokes. The mean ages of the IHD and stroke patients were respectively 57 and 59 years $(\mathrm{P}=0.09)$. As a cohort study, we used the person-year indicator for calculating the incidence of CVD events. The incidence of IHD and stroke increased with the increasing age of the 
participants. Table 1 shows the incidence rates of IHD and stroke according to age groups. Accordingly, the incidence of IHD and stroke in the subjects aged 35 - 44 years were 481.4 (95\% CI, 397.9 - 582.4) and 98.6 per 100,000 person years (95\% CI, 64.9 -149.7), respectively. Table 1 showed an increase of $57.7 \%$ in IHD incidence and 57.9\% in stroke incidence in the 45- to 54-year age group, in comparison with the 35- to 44-year age group. The incidences of IHD and stroke based on the patients' age range show that stroke follows a slighter slope relative to IHD.

We calculated the cumulative percent of CVD events based on age group among all the patients who devel- oped the disease during the 10-year follow-up. It was $27.4 \%$ in the patients aged less than 55 years and $53.2 \%$ in those aged less than 65 years. Differences in the frequency of CVD risk factors in those who developed IHD or stroke were significant in terms of FBG level, diabetes, and serum levels of total cholesterol and LDL-C (Table 2).

Table 3 shows the adjusted RRs of the CVD risk factors for the occurrence of IHD and stroke, and the difference between them presented as RRR. Accordingly, IHD and stroke had RRs of 1.90 (95\% CI, $1.59-2.26$ ) and 1.76 (95\% CI, 1.11 - 2.81) in the patients with hypertension, but RR did not significantly different between them $(P=0.76)$.

\begin{tabular}{|c|c|c|c|c|c|c|}
\hline \multirow[t]{2}{*}{ Age, $y$} & \multicolumn{3}{|c|}{ IHD } & \multicolumn{3}{|c|}{ Stroke } \\
\hline & Person-Years & Rate & 95\% CI & Person-Years & Rate & 95\% CI \\
\hline $35-44$ & 22016 & 481.4 & 397.9 - 582.4 & 22309 & 98.6 & $64.93-149.76$ \\
\hline $45-54$ & 12819 & 1138.8 & $968.3-1339.4$ & 13254 & 233.8 & $164.48-332.57$ \\
\hline $55-64$ & 8689 & 1714.6 & $1460.2-2013.1$ & 9064 & 408.1 & $295.75-563.37$ \\
\hline $65-74$ & 5366 & 2534.3 & 2142.2 - 2998.1 & 5672 & 669.9 & $487.46-920.67$ \\
\hline$\geq 75$ & 1109 & 2342.7 & $1595.1-3440.8$ & 1148 & 1131.4 & $656.98-1948.67$ \\
\hline Total & 50002 & 1125.9 & $1036.6-1222.9$ & 51449 & 274 & $232.35-323.23$ \\
\hline
\end{tabular}

a Abbreviation: IHD, ischemic heart disease.

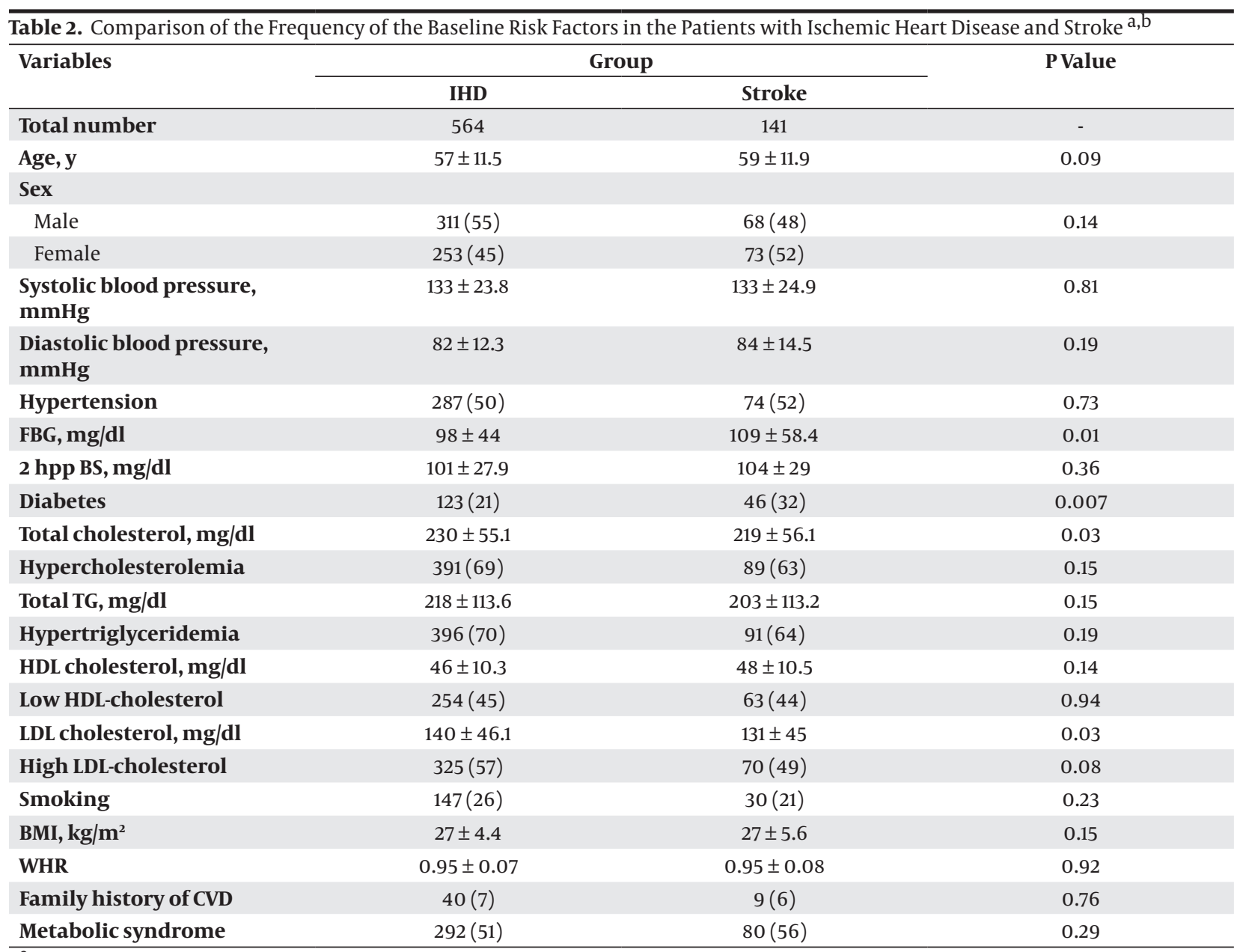

\footnotetext{
${ }^{\mathrm{a}}$ Data are expressed as mean \pm SD for continuous variables and number (percentage) for categorical variables.
}

b Abbreviation: CVD, cardiovascular disease; FBG, fasting blood glucose; IHD, ischemic heart disease; TG, triglyceride; WHR, waist-to-hip ratio. 
Aalami Harandi S et al.

\begin{tabular}{|c|c|c|c|c|c|}
\hline \multirow[t]{3}{*}{ Risk Factors } & \multicolumn{4}{|c|}{ Group } & \multirow[t]{3}{*}{ P Value $^{\text {b }}$} \\
\hline & \multicolumn{2}{|c|}{ IHD } & \multicolumn{2}{|c|}{ Stroke } & \\
\hline & Adjusted ${ }^{b}$ RR & 95\% CI & Adjusted ${ }^{c}$ RR & 95\% CI & \\
\hline Hypertension & 1.90 & $1.59-2.26$ & 1.76 & $1.11-2.81$ & 0.762 \\
\hline Diabetes & 1.94 & $1.61-2.35$ & 3.26 & $2.23-4.76$ & 0.016 \\
\hline Hypercholesterolemia & 1.47 & $1.22-1.77$ & 1.03 & $0.72-1.56$ & 0.104 \\
\hline Hypertriglyceridemia & 1.63 & $1.36-1.96$ & 1.36 & $0.91-2.03$ & 0.420 \\
\hline Low HDL-cholesterol & 1.24 & $1.03-1.48$ & 1.23 & $0.76-1.97$ & 0.975 \\
\hline High LDL-cholesterol & 1.40 & $1.19-1.65$ & 0.92 & $0.63-1.34$ & 0.045 \\
\hline Current smoking & 1.17 & $0.94-1.44$ & 0.80 & $0.42-1.51$ & 0.269 \\
\hline Obesity & 1.15 & $0.94-1.41$ & 1.23 & $0.82-1.88$ & 0.775 \\
\hline High WHR & 1.68 & $1.35-2.09$ & 1.52 & $0.91-2.54$ & 0.725 \\
\hline Family history of CVD & 1.64 & $1.22-2.21$ & 1.46 & $0.69-3.08$ & 0.777 \\
\hline Metabolic syndrome & 1.88 & $1.58-2.24$ & 2.25 & $1.49-3.40$ & 0.431 \\
\hline
\end{tabular}

a Abbreviation: CVD, cardiovascular disease; IHD, ischemic heart disease; WHR, waist-to-hip ratio.

$\mathrm{b}$ Ratio of relative risks was calculated for comparing two relative risks.

C Adjusted based on sex and age.

In contrast, the RRs for IHD and stroke in the patients with diabetes were statistically different (1.94 and 3.26, respectively; $\mathrm{P}=0.016$ ). Furthermore, the RR of high LDLC was significantly higher for IHD than for stroke (1.40 vs. $0.92, \mathrm{P}=0.045)$.

The RRs of the other factors, including hypercholesterolemia, hypertriglyceridemia, low HDL-C, smoking, obesity, waist circumference, family history, and metabolic syndrome were not significantly different between the study groups.

\section{Discussion}

This study presents a comparison between the RRs of cardiometabolic risk factors of CVDs, mainly IHD and stroke. According to the results, hypertension and diabetes mellitus were the most effective risk factors of IHD. Similarly, diabetes mellitus, metabolic syndrome, and hypertension were the most effective risk factors of stroke, but the effect of diabetes mellitus on stroke was more significant than that on IHD. Furthermore, the risk of developing stroke among the diabetes patients was relatively twice as high as that among IHD patients. Another finding was that the RR of high LDL-C was significantly higher for IHD than for stroke.

Another interesting result in the present study is that hypertension has equal RRs for the occurrence of both IHD and stroke. Previous reports indicated that hypertension (as a modifiable risk factor) has more potent association with the risk of stroke rather than IHD. However, according to our findings, hypertension has a strong association with the risk of IHD as well ( $R R=1.90$; 95\% CI, 1.59-2.26). These findings are similar to those of our previous study (18) and those of other studies such the Honolulu Heart Program that showed that systolic blood pressure and smoking were the most important risk factors of IHD, rather than other risk factors, including high cholesterol level (23). In Iran, the prevalence of hypertension is high, with an increasing trend in recent years (24). Unfortunately, its control level among treated patients remains low (25-27). This may be one explanation of the high prevalences of IHD and stroke, and their occurrences among younger patients in Iran. The RRs of the other factors such as smoking, obesity, and metabolic syndrome were not significantly different between the two groups of patients.

A study conducted in the United States in 2005 among 18,662 men revealed that the increase in the incidence of stroke related to hypertension was similar to IHD. The RRs of hypertension for IHD and stroke were 1.74 and 1.90, respectively, with no significant difference $(P=0.36)$, as in our results. Diabetes has the highest RR for both IHD and stroke, but the difference in RR was not significant (2.31 vs. 2.64, $\mathrm{P}=0.44$ ). Furthermore, it showed that the incidences of stroke and coronary heart disease (CHD) increase 28 and 13 times, respectively, with increasing age (28). However, in our study, we observed a lower outbreak of stroke with increasing age, as compared with cardiovascular disease.

Other studies showed that cigarette smoking is the most important modifiable risk factor of CHD (29), and the continuous smoking hazard ratio for CHD is 2.9 (95\% CI, 2.7 - 3.1). However, this was not the case in our study, which may be due to higher prevalence of cigarette smoking in Western communities than in nations such 
Aalami Harandi S et al.

as Iran (19\% vs. $9.9 \%$ in 2011$)(29,30)$.

Some studies considered a major role for hypercholesterolemia in CHD (17), and LDL-C has the best established link with the incidence of MI and cardiovascular death (26). This is different from our results that showed that diabetes mellitus and hypertension had the highest RR for IHD. The reason may be related to the dietary habits and lifestyle of our study population. Our previous studies showed that the Iranian lipid profile differs from that of Western populations, mostly in regard to lower mean levels of total cholesterol and LDL-C but higher TG levels and more prevalent low HDL-C level (31).

A prospective study in Japan in 2007 revealed that metabolic syndrome is interrelated with a higher IHD risk. In addition, IHD patients have an older mean age and hypertension, hypercholesterolemia, hypertriglyceridemia, smoking, and lower HDL-C level than the healthy people. These factors were identical between the males and females. Stroke patients have an older mean age and higher hypertension than the control group. High level of smoking and low level of HDL-C were observed more frequently among the men. This study demonstrated that all components of metabolic syndrome, except for glucose disturbance, can increase the risk of IHD two times more among males. However, among females, they were not significantly interrelated with hypertension and obesity. In addition, stroke risk increases 3 or 4 times by hypertension and 1.5-2 times by other components of metabolic syndrome in men. This relationship was observed in females as well, except for HDL-C level. Moreover, this study showed a direct relationship between stroke and IHD risk, and the number of metabolic syndrome components (32). Other report from Japan showed that metabolic syndrome was related with two-fold higher risks of IHD and stroke, and total CVD (33).

In 2002, a prospective study from the Copenhagen City Heart study of 12,000 men and women aged 30-79 years reported a higher RR of diabetes mellitus for IHD than for other risk factors (RR of 1.69 for men and 2.74 for women), as shown in our study. The RR of hypertension for IHD was 1.46 for men and 1.42 for women (34). In addition, a number of Asian studies showed that hypertriglyceridemia and hypertension, and low HDL-C are important risk factors of IHD and diabetes mellitus, and that hypertension and low HDL-C level are the main risk factors of stroke (35-39). Perhaps the difference in RR of cardiovascular risk factors may be related to different prevalence in different populations. The interference of other factors such as genetics, difference in age groups, and other factors not considered in these studies should be considered before any conclusion.

According to our results, approximately one third (27\%) of all CVD events occurred among patients younger than 55 years, which highlights the high frequency of premature CVD in Iran. When the cutoff age was considered as 65 years or younger, more than half of the events were within this definition. We consider this to be among our important secondary findings that need more attention by health authorities. The younger patients have different risk factor profiles and prognoses from those of older patients. Therefore, health policies should consider these findings in CVD screening, diagnosis, and treatment strategies, and should intensify modifying the population lifestyle toward a healthy one starting from childhood in order to reduce cardiometabolic risk factors.

The strength of our study is its large sample size, including participants from urban and rural areas from central Iran; our standard measurement of variables; and confirming the CVD events by an expert panel of 3cardiologits and 1 neurologist. While the limitations include noncoverage of the information of other parts of Iran and an inherent selection bias because of the observational nature of the study design.

In conclusion, according to our study findings, the RR of diabetes for stroke was significantly higher than the $R R$ for IHD, and the RR of high LDL-C for IHD was significantly higher than the RR for stroke. Meanwhile, the RR of hypertension did not differ between IHD and stroke. Other cardiometabolic factors have no significant differences and have the same effect on both diseases. Thus, patients with diabetes mellitus and high LDL-C should be treated accurately and intensively evaluated for other risk factors of stroke and IHD. The great effect of hypertension on the occurrence of IHD and stroke should be taken seriously by health decision makers, as the prevalence of hypertension and the frequency of uncontrolled hypertension is high in our country.

\section{Acknowledgements}

This cohort study was conducted by the Isfahan cardiovascular research institute (ICRI), which is affiliated with the Isfahan university of medical sciences. The authors thank the ICS team in ICRI, Isfahan provincial health center, Najaf-Abad health office, and Arak University of Medical Sciences. We particularly wish to acknowledge Dr. Mohamad Kermani Alghoerishi, Isfahan university of medical sciences, who had provided important comments.

\section{Authors' Contributions}

Study concept and design: Sarrafzadegan and Sadeghi. Analysis and interpretation of data: Sarrafzadegan and Talaei. Drafting of the manuscript: Aalami Harandi. Critical revision of the manuscript for important intellectual content: Sarrafzadegan. Statistical analysis: Dianatkhah. Administrative, technical, and material support: Aalami Harandi, Pourmoghaddas, Salehi, and Sedighifard. Study supervision: Sarrafzadegan.

\section{Funding/Support}

This study was supported by the Isfahan cardiovascular research institute (ICRI), which is affiliated with the Isfahan university of medical sciences. 


\section{References}

1. Tunstall-Pedoe H. Preventing Chronic Diseases. A Vital Investment: WHO Global Report. Geneva: World Health Organization, 2005. pp 200. CHF 30.00. ISBN 924 1563001. Also published on http://www. who. int/chp/chronic_disease_report/en. Int J Epidemiol. 2006;35(4):1107.

2. Motlagh B, O'Donnell M, Yusuf S. Prevalence of cardiovascular risk factors in the Middle East: a systematic review. Eur J Cardiovasc Prev Rehabil. 2009;16(3):268-80.

3. Talaei M, Sadeghi M, Marshall T, Thomas GN, Iranipour R, Nazarat $\mathrm{N}$, et al. Anthropometric indices predicting incident type 2 diabetes in an Iranian population: the Isfahan Cohort Study. Diabetes Metab. 2013;39(5):424-31.

4. Talaei M, Sadeghi M, Mohammadifard N, Shokouh P, Oveisgharan S, Sarrafzadegan N. Incident hypertension and its predictors: the Isfahan Cohort Study. J Hypertens. 2014;32(1):30-8.

5. Talaei M, Sarrafzadegan N, Sadeghi M, Oveisgharan S, Marshall T, Thomas GN, et al. Incidence of cardiovascular diseases in an Iranian population: the Isfahan Cohort Study. Arch Iran Med. 2013;16(3):138-44.

6. Goldstein LB, Bushnell CD, Adams RJ, Appel LJ, Braun LT, Chaturvedi S, et al. Guidelines for the primary prevention of stroke: a guideline for healthcare professionals from the American Heart Association/American Stroke Association. Stroke. 2011;42(2):517-84.

7. Wilson PW, D'Agostino RB, Levy D, Belanger AM, Silbershatz H, Kannel WB. Prediction of coronary heart disease using risk factor categories. Circulation.1998;97(18):1837-47.

8. Wolf PA, D'Agostino RB, Belanger AJ, Kannel WB. Probability of stroke: a risk profile from the Framingham Study. Stroke. 1991;22(3):312-8.

9. Hubert HB, Feinleib M, McNamara PM, Castelli WP. Obesity as an independent risk factor for cardiovascular disease: a 26-year follow-up of participants in the Framingham Heart Study. Circulation. 1983;67(5):968-77.

10. Kannel WB, McGee DL. Diabetes and cardiovascular disease: the Framingham study. JAMA. 1979;241(19):2035-8.

11. McBride PE. The health consequences of smoking. Cardiovascular diseases. Med Clin North Am. 1992;76(2):333-53.

12. Rodgers A, MacMahon S. Blood pressure and the global burden of cardiovascular disease. Clin Exp Hypertens. 1999;21(5-6):543-52.

13. Sadeghi M, Talaei M, Parvaresh Rizi E, Dianatkhah M, Oveisgharan S, Sarrafzadegan N. Determinants of incident prediabetes and type 2 diabetes in a 7-year cohort in a developing country: The Isfahan Cohort Study 72. J Diabetes. 2015;7(5):633-41.

14. Sarrafzadegan N, Gharipour M, Sadeghi M, Khosravi AR, Tavassoli AA. Metabolic syndrome in Iranian elderly. ARYA Atheroscler 2012;7(4):157-61.

15. Kannel WB, D'Agostino RB, Sullivan L, Wilson PW. Concept and usefulness of cardiovascular risk profiles. Am Heart $J$. 2004;148(1):16-26.

16. Shimamoto T, Komachi Y, Inada H, Doi M, Iso H, Sato S, et al. Trends for coronary heart disease and stroke and their risk factors in Japan. Circulation. 1989;79(3):503-15.

17. Stokes J3, Kannel WB, Wolf PA, Cupples LA, D'Agostino RB. The relative importance of selected risk factors for various manifestations of cardiovascular disease among men and women from 35 to 64 years old: 30 years of follow-up in the Framingham Study. Circulation. 1987;75(6 Pt 2):V65-73.

18. Sarrafzadegan N, Talaei M, Sadeghi M, Kelishadi R, Oveisgharan S, Mohammadifard N, et al. The Isfahan cohort study: rationale, methods and main findings. J Hum Hypertens. 2011;25(9):545-53.

19. Sarraf-Zadegan N, Sadri G, Malek Afzali H, Baghaei M, Mohammadi Fard N, Shahrokhi S, et al. Isfahan Healthy Heart Programme: a comprehensive integrated community-based programme for cardiovascular disease prevention and control. Design, methods and initial experience. Acta Cardiol. 2003;58(4):309-20.

20. Expert Panel on Detection. Executive Summary of the Third Report of the National Cholesterol Education Program (NCEP) Expert Panel on Detection, Evaluation, and Treatment of High
Blood Cholesterol in Adults (Adult Treatment Panel III). J Am Med Assoc. 2001;285(19):2486-97.

21. Altman DG, Bland JM. Interaction revisited: the difference between two estimates. BMJ. 2003;326(7382):219.

22. Kleinman LC, Norton EC. What's the Risk? A simple approach for estimating adjusted risk measures from nonlinear models including logistic regression. Health Serv Res. 2009;44(1):288-302.

23. Yano K, Reed DM, McGee DL. Ten-year incidence of coronary heart disease in the Honolulu Heart Program. Relationship to biologic and lifestyle characteristics. Am J Epidemiol. 1984;119(5):653-66.

24. Khosravi A, Pourmoghaddas M, Kelishadi R, Sabet B, Ansari R, Shirani S. Trends in Blood Pressure Level, Prevalence of Hypertention and its Care in Isfahan. Iranian Red Crescent Med J. 2005;2006(2):62-8.

25. Gee ME, Campbell N, Sarrafzadegan N, Jafar T, Khalsa TK, Mangat $\mathrm{B}$, et al. Standards for the uniform reporting of hypertension in adults using population survey data: recommendations from the World Hypertension League Expert Committee. J Clin Hypertens (Greenwich). 2014;16(11):773-81.

26. Khosravi A, Ansari R, Shirani S, Baghaei AM. Causes of uncontrolled Blood Pressure in the Over-65 population 2010. ARYA Atheroscler. 2010;1(2)

27. Khosravi A, Pourheidar B, Roohafza H, Moezzi M, Mousavi M, Hajiannejad A, et al. Evaluating factors associated with uncontrolled hypertension: Isfahan cohort study, Iran. ARYA Atheroscler 2014;10(6):311-8.

28. Glynn RJ, Rosner B. Comparison of risk factors for the competing risks of coronary heart disease, stroke, and venous thromboembolism. Am J Epidemiol. 2005;162(10):975-82.

29. Lilly LS. Braunwald's Heart Disease: A Textbook of Cardiovascular Medicine. Elsevier - Health Sciences Division; 2012.

30. Roohafza H, Sadeghi M, Shahnam M, Bahonar A, Sarafzadegan N Perceived factors related to cigarette and waterpipe (ghelyan) initiation and maintenance in university students of Iran. Int J Public Health. 2011;56(2):175-80.

31. Solhpour A, Parkhideh S, Sarrafzadegan N, Asgary S, Williams K, Jungner I, et al. Levels of lipids and apolipoproteins in three cultures. Atherosclerosis. 2009;207(1):200-7.

32. Iso H, Sato S, Kitamura A, Imano H, Kiyama M, Yamagishi K, et al. Metabolic syndrome and the risk of ischemic heart disease and stroke among Japanese men and women. Stroke. 2007;38(6):1744-51.

33. Chei CL, Yamagishi K, Tanigawa T, Kitamura A, Imano H, Kiyama $\mathrm{M}$, et al. Metabolic Syndrome and the Risk of Ischemic Heart Disease and Stroke among Middle-Aged Japanese. Hypertens Res. 2008;31(10):1887-94.

34. Schnohr P, Jensen JS, Scharling H, Nordestgaard BG. Coronary heart disease risk factors ranked by importance for the individual and community. A 21 year follow-up of 12000 men and women from The Copenhagen City Heart Study. Eur Heart J. 2002;23(8):620-6.

35. Iso H, Imano H, Kitamura A, Sato S, Naito Y, Tanigawa T, et al. Type 2 diabetes and risk of non-embolic ischaemic stroke in Japanese men and women. Diabetologia. 2004;47(12):2137-44.

36. Soyama Y, Miura K, Morikawa Y, Nishijo M, Nakanishi Y, Naruse $\mathrm{Y}$, et al. High-density lipoprotein cholesterol and risk of stroke in Japanese men and women: the Oyabe Study. Stroke. 2003;34(4):863-8.

37. Iso H, Naito Y, Sato S, Kitamura A, Okamura T, Sankai T, et al. Serum triglycerides and risk of coronary heart disease among Japanese men and women. Am J Epidemiol. 2001;153(5):490-9.

38. Kitamura A, Iso $H$, Naito Y, Iida M, Konishi M, Folsom AR, et al. High-density lipoprotein cholesterol and premature coronary heart disease in urban Japanese men. Circulation. 1994;89(6):2533-9.

39. Shimozato M, Nakayama T, Yokoyama T, Yoshi-ike N, Yamaguchi M, Date C. A 15.5-year cohort study on risk factors for possible myocardial infarction and sudden death within 24 hours in a rural Japanese community.J Epidemiol. 1996;6(1):15-22. 